\title{
Physical Structure of the Planetary Nebula NGC 3242 from the Hot Bubble to the Nebular Envelope ${ }^{1}$
}

\author{
Nieves Ruiz ${ }^{1}$, Martín A. Guerrero ${ }^{1}$ \\ ${ }^{1}$ Instituto de Astrofísica de Andalucía, CSIC, Granada 18008, Spain \\ nieves@iaa.es, mar@iaa.es \\ and \\ You-Hua $\mathrm{Chu}^{2}$, Robert A. Gruendl ${ }^{2}$ \\ 2 Astronomy Department, University of Illinois at Urbana-Champaign, Urbana, IL 61801 \\ yhchu@astro.illinois.edu, gruendl@astro.illinois.edu
}

\begin{abstract}
One key feature of the interacting stellar winds model of the formation of planetary nebulae (PNe) is the presence of shock-heated stellar wind confined in the central cavities of PNe. This so-called hot bubble should be detectable in X-rays. Here we present XMM-Newton observations of NGC 3242, a multiple-shell PN whose shell morphology is consistent with the interacting stellar winds model. Diffuse X-ray emission is detected within its inner shell with a plasma temperature $\sim 2.35 \times 10^{6} \mathrm{~K}$ and an intrinsic X-ray luminosity $\sim 2 \times 10^{30} \mathrm{ergs} \mathrm{s}^{-1}$ at the adopted distance of 0.55 kpc. The observed X-ray temperature and luminosity are in agreement with "ad-hoc" predictions of models including heat conduction. However, the chemical abundances of the X-ray-emitting plasma seem to imply little evaporation of cold material into the hot bubble, whereas the thermal pressure of the hot gas is unlikely to drive the nebular expansion as it is lower than that of the inner shell rim. These inconsistencies are compounded by the apparent large filling factor of the hot gas within the central cavity of NGC 3242 .
\end{abstract}

Subject headings: planetary nebulae: individual (NGC 3242)

\section{Introduction}

Planetary Nebulae (PNe) consist of stellar material ejected by low- and intermediate-mass stars $\left(0.8-1.0 \mathrm{M}_{\odot} \leq M_{i} \leq 8-10 \mathrm{M}_{\odot}\right)$. Towards the end of the Asymptotic Giant Branch (AGB), these stars experience copious mass loss and eject most of their stellar envelope through a slow, dense AGB wind. The ejected material is subsequently ionized by the central star and becomes a PN. PNe eventually disperse into the interstellar medium as they expand, whereas the stellar cores, mainly composed of carbon and oxygen, will evolve to-

\footnotetext{
${ }^{1}$ Based on observations obtained with XMM-Newton, an ESA science mission with instruments and contributions directly funded by ESA Member States and NASA.
}

ward the white dwarf stage.

Near the time when the hot stellar core is exposed, the slow AGB wind, with terminal velocities $5-30 \mathrm{~km} \mathrm{~s}^{-1}$ (Eder et al. 1988), is superseded by a fast stellar wind with terminal velocities 1,000-4,000 $\mathrm{km} \mathrm{s}^{-1}$ (Cerruti-Sola \& Perinotto 1985; Guerrero et al. 2010). This fast stellar wind sweeps up the slower AGB wind to form a PN (Kwok 1983). In this interacting stellar winds (ISW) model, the physical structure of a PN would be similar to that of a wind-blown bubble, as modeled by Weaver et al. (1977), comprising a central cavity filled with shocked fast wind (the so-called hot bubble), a dense shell of swept-up AGB wind at $10^{4} \mathrm{~K}$, and an outer envelope of unperturbed expanding AGB wind. In a simplistic model, the 
temperature of the shocked stellar wind inside the hot bubble would be $10^{7}-10^{8} \mathrm{~K}$, but turbulent mixing (e.g., Mellema \& Frank 1995) or heat conduction (Zhekov \& Perinotto 1998; Steffen et al. 2008) lowers the temperature of the hot gas to $10^{6}-10^{7} \mathrm{~K}$ and raises its density to produce optimal conditions for the emission of soft X-rays. Therefore, X-ray observations of shock-heated hot gas in PNe provide us a direct means to examine the interaction of the fast stellar wind with the AGB wind and to investigate the transfer of energy and momentum to the PN envelope.

ROSAT observations showed hints of diffuse Xray emission in a few PNe (Guerrero et al. 2000). However, it was not until the advent of Chandra and XMM-Newton, with their unprecedented resolution and sensitivity, that we were finally able to unambiguously detect hot gas in PNe. Chandra and XMM-Newton have resolved the diffuse X-ray emission in a handful of PNe (e.g., Kastner et al. 2000, 2001; Chu et al. 2001; Guerrero et al. 2002, 2005) and revealed unexpected, hard X-ray emission from the central stars of several PNe that may originate from the coronal emission of unseen faint binary companions or shocks within the fast stellar winds (Guerrero et al. 2001; Kastner et al. 2003; Montez et al. 2010). Observations of diffuse X-ray emission in PNe demonstrate that hot gas in elliptical PNe is confined within the innermost nebular shell and that the high pressure of the hot gas may indeed drive the nebular expansion as expected in bubble models.

The observed values of $L_{\mathrm{X}}$ and $T_{\mathrm{X}}$ are in agreement with predictions of the time-dependent models developed by Steffen et al. (2008) that include heat-conduction for PNe with central stars of normal, hydrogen-rich surface composition. There is, however, an unsolved puzzle: the analyses of the chemical composition of the X-ray-emitting plasma in PNe suggest that it is mainly composed of stellar wind, with little contamination of material from the cold nebular shell (e.g., NGC 2392 and NGC 6543, Guerrero et al. 2005; Chu et al. 2001). This discrepancy has been further illustrated by the analysis of the high-spectral resolution Chandra LETG spectrum of $\mathrm{BD}+30^{\circ} 3639$ that conclusively confirms the stellar wind composition of its X-ray-emitting plasma ( $\mathrm{Yu}$ et al. 2009). This problem has prompted alternative mechanisms for the production of hot gas in PNe, including the action of fast collimated outflows and/or slow fragments in the onset of the fast stellar wind (Soker \& Kastner 2003), and the absorption of energy from the stellar wind by slowly moving ions embedded in the wind itself, the so-called pick-up ions (Soker et al. 2010).

NGC 3242 (PNG261.0+32.0), the Ghost of Jupiter, is a multiple-shell PN with a bright, $28^{\prime \prime} \times 20^{\prime \prime}$ inner ellipsoidal shell and ansae surrounded by a fainter, $46^{\prime \prime} \times 40^{\prime \prime}$ moderately elliptical envelope. These two shells are further enclosed by arcs and a giant broken halo revealed by deep images (Corradi et al. 2003, 2004). The double-shell morphology of the main nebula of NGC 3242 is highly suggestive of interactions between the fast stellar wind of its central star $\left(v_{\infty}=2,400 \mathrm{~km} \mathrm{~s}^{-1}\right.$, Pauldrach et al. 2004) and the previous slow AGB wind. The AGB wind (the nebular envelope) has been swept by the fast stellar wind to form a thin ionized shell with a central cavity that can be expected to be filled with shocked fast wind. This shockheated gas should emit X-rays, and the diffuse Xray emission from NGC 3242 is likely detectable because of its proximity (distance $=0.55 \pm 0.23$ kpc, Terzian 1997; Mellema 2004), and low extinction (Balick et al. 1993; Henry et al. 2000; Pottasch \& Bernard-Salas 2008).

In this paper, we present XMM-Newton observations of NGC 3242 that have detected diffuse Xray emission within its innermost nebular shell. The observations are described in $\S 2$, the results are presented in $\S 3$, the physical structure of the optical nebula is investigated in $\S 4$, and the effects of the shocked stellar wind in the nebula are discussed in $\S 5$.

\section{Observations}

\subsection{XMM-Newton X-Ray Observations}

NGC 3242 was observed with the XMM-Newton Observatory in Revolution 730 on 2003 December 4 using the EPIC-MOS1, EPIC-MOS2, and EPICpn CCD cameras (OBSID $=0200240401)$. The two EPIC-MOS cameras were operated in the FullFrame Mode for a total exposure time of $19.1 \mathrm{ks}$, while the EPIC-pn camera was operated in the Extended Full Frame Mode for a total exposure time of $15.7 \mathrm{ks}$. The Medium filter was used for all observations. The XMM-Newton products were 
processed using the XMM-Newton Science Analysis Software (SAS version 10.0.0) and the calibration files from the Calibration Access Layer available on 2010 September 16. The event files were screened to eliminate events due to charged particles or associated with periods of high background. For the EPIC-MOS observations, only events with CCD patterns 0-12 were selected; for the EPICpn observation, only events with CCD pattern 0 (single pixel events) were selected. Time intervals of high background, when the count rate in the background dominated $10-12 \mathrm{keV}$ energy range is $\geq 0.3 \mathrm{cnts} \mathrm{s}^{-1}$ for EPIC-MOS and $\geq 1.4 \mathrm{cnts} \mathrm{s}^{-1}$ for EPIC-pn, were discarded. The resulting net exposure times are $18.7 \mathrm{ks}, 18.7 \mathrm{ks}$, and $13.6 \mathrm{ks}$ for the EPIC-MOS1, EPIC-MOS2, and EPIC-pn observations, respectively.

The XMM-Newton EPIC observations detect a source of diffuse X-ray emission at the location of NGC 3242. An inspection of EPIC-pn and EPICMOS images at different energy ranges reveals that this source is soft, with most emission below $1.0 \mathrm{keV}$ and very little emission at higher energies. The EPIC-pn background-subtracted count rate in the $0.38-2.0 \mathrm{keV}$ energy range is $31.3 \pm 1.6$ cnts ks ${ }^{-1}$ for a total of $422 \pm 22$ counts. The EPICpn net count rates in the energy ranges $0.38-1.0$ $\mathrm{keV}$ and $1.0-2.0 \mathrm{keV}$ are $30.3 \pm 1.6 \mathrm{cnts} \mathrm{ks}^{-1}$, and $1.1 \pm 0.4 \mathrm{cnts} \mathrm{ks}^{-1}$, respectively. The EPIC-MOS background-subtracted count rates in the 0.38-2.0 $\mathrm{keV}$ energy band are $4.4 \pm 0.5 \mathrm{cnts} \mathrm{ks}^{-1}$ for MOS1 and $4.8 \pm 0.5 \mathrm{cnts} \mathrm{ks}^{-1}$ for MOS2, with a total of $83 \pm 10 \mathrm{cnts}$ for MOS1 and $90 \pm 10 \mathrm{cnts}$ for MOS2.

\subsection{Archival Narrow-band HST Imaging}

In order to examine the spatial correlation between the diffuse X-ray emission and the optical nebula, narrow-band WFPC2 images of NGC 3242 in the $\mathrm{H} \alpha$, He II $\lambda 4686$, [N II] $\lambda 6583$, and [O III] $\lambda 5007$ emission lines were retrieved from the HST archive (Proposal ID 7501 and 8773, PI: Arsen Hajian, and Proposal ID 6117, PI: Bruce Balick). The images used in this work are listed in Table1 with their integration times and filters. In all cases, the innermost shell of the nebula was registered on the WFPC2-PC1 CCD, while a fraction of the outer envelope was missed by the detector. These images were calibrated via the pipeline procedure and cosmic rays were removed by combining different exposures obtained with the same filter using standard IRAF2 routines. The final images have total exposure times of $100 \mathrm{~s}$ for $\mathrm{H} \alpha$, $160 \mathrm{~s}$ for He II, $1260 \mathrm{~s}$ for [O III], and $2840 \mathrm{~s}$ for [N II].

\subsection{Medium-Dispersion Spectroscopy Ob- servations}

Medium-dispersion long-slit spectroscopic observations of NGC 3242, obtained using the GoldCam CCD Spectrograph (GCCAM) on the $2.1 \mathrm{~m}$ telescope at the Kitt Peak National Observatory (KPNO) on 1996 December 7, were kindly provided to us by Dr. K. Kwitter (Henry et al. 2000). The observations, covering the spectral region $3600-9600 \AA$, were obtained using a $285^{\prime \prime} \times 5^{\prime \prime}$ slit oriented along the East-West direction and centered at $8^{\prime \prime}$ south of the central star. The spatial scale of the observations is $0 . \prime 78 \mathrm{pix}^{-1}$. The grating 240 was used with the GG-345 blocking filter to cover the blue spectral region $3650-6750 \AA$ at a spectral dispersion $1.49 \AA$ pix $^{-1}$, whereas the red spectral region $5650-9600 \AA$ was observed at a spectral dispersion $1.86 \AA \mathrm{pix}^{-1}$ using grating 58 with the OG-530 blocking filter. Two exposures of $60 \mathrm{~s}$ each were acquired in the blue spectral range, while exposures of $60 \mathrm{~s}, 30 \mathrm{~s}$, and two of $20 \mathrm{~s}$ were acquired in the red spectral range. The original spectra were reduced and analyzed using standard IRAF routines. For the flux calibration we used several observations of the spectrophotometric standard stars $\mathrm{BD}+28^{\circ} 4211$ and Hiltner 600 obtained on the same night.

\section{XMM-Newton Data Analysis}

\subsection{Spatial Properties of the X-ray Emis- sion from NGC 3242}

To study the spatial distribution of the X-ray emission from NGC 3242, we have produced EPIC images of NGC 3242 in the energy band $0.25-2.5$ $\mathrm{keV}$ by extracting the individual EPIC-pn, EPICMOS1, and EPIC-MOS2 images, mosaicing them together, applying the exposure map correction, and smoothing it. The raw image is shown in the

\footnotetext{
2 IRAF, the Image Reduction and Analysis Facility, is distributed by the National Optical Astronomy Observatory, which is operated by the Association of Universities for Research in Astronomy (AURA) under cooperative agreement with the National Science Foundation.
} 
left panel of Figure1, while the exposure map corrected, smoothed version of the image overlaid by $\mathrm{X}$-ray contours is shown in the central panel.

To compare the relative spatial distribution of the X-ray-emitting gas and the ionized nebular material, we have superposed the X-ray contours on the HST WFPC2 [O III] image of NGC 3242 (Fig. 1 right). The comparison between the Xray contours and the optical image suggests that the X-ray-emitting gas is confined within the innermost shell of NGC 3242, as the location of the lowest intensity level X-ray contours outside the $28^{\prime \prime} \times 20^{\prime \prime}$ inner shell of NGC 3242 is most likely caused by the point-spread function (PSF) of XMM-Newton that is known to have a half energy width (HEW) of $15^{\prime \prime} 2$ for EPIC-pn and $133^{\prime \prime} 0$ $13^{\prime \prime} 8$ for EPIC-MOS and significantly extended wings.

The X-ray image and contours also suggest that the detailed morphology of the X-ray emission is asymmetric, with an emission peak northwest of the central star. We further compare in Figure 2 the surface brightness profile of the X-ray emission of NGC 3242 along the major and minor nebular axes with the PSF from a point source in the field of view. Both profiles of the nebular emission are asymmetric and more extended than those of the point-source, more clearly for the profile along the major axis of the nebula.

In order to better understand the distribution of X-ray-emitting gas in NGC 3242 we have constructed simulated observations for comparison with the XMM-Newton images. For these simulated observations we modeled the emission from NGC 3242 as though it arose from a constantdensity ellipsoidal shell of X-ray-emitting gas interior to the innermost nebular shell. We assumed a prolate ellipsoid with semi-major and semi-minor axes of $11^{\prime \prime} .25 \times 7^{\prime \prime} .5$ in the plane of the sky. Three models were considered: Model A assumed the $\mathrm{X}$-ray-emitting gas was confined to an ellipsoidal shell with a fractional width $(\Delta r / r)$ of 0.1 ; Model $\mathrm{B}$ assumed a similar shell with a fractional width of 0.2 ; and Model $\mathrm{C}$ assumed the X-ray-emitting gas filled the ellipsoid with constant density.

To simulate yhe XMM-Newton observations, we generated random simulated $\mathrm{X}$-rays based on the model distribution of the X-ray emitting gas with further randomization in the plane of the sky consistent with the $X M M-N e w t o n \mathrm{PSF}^{3}$. We also added random X-ray events to the simulated image to mimic the background emission. The total number of simulated source and noise counts were set to match those of the actual XMM-Newton observations. Each model was used to make ten simulations to explore the variations caused by the small number statistics of these Monte Carlo simulations. We then adaptively smoothed the simulated observations with the same parameters used for the actual observations. Figure 3 shows a typical realization for each of the three models described. In the realizations of models A and B (the shell models) a central deficit in X-ray emission is always apparent. In the realizations of Model C the X-ray emission peaks on or near the center; the example shown in Fig. [3 even exhibited an offset peak matching the actual observation. These simulated observations demonstrate that the diffuse X-ray emission from NGC 3242 is more consistent with that from a central cavity filled with Xray-emitting gas than from a thin ellipsoidal shell. They also suggest that the asymmetric distribution of the X-ray emission may be spurius due to the low count number.

\subsection{Spectral Properties of the X-ray Emission from NGC 3242}

To study the spectral properties of the Xray emission from NGC 3242, we have extracted its EPIC-pn, EPIC-MOS1, and EPIC-MOS2 background-subtracted spectra (Figure 4). Our description of the spectral properties of NGC 3242 will focus on the EPIC-pn spectrum as the number of counts in this spectrum is $\sim 5$ times larger than in the EPIC-MOS1 and EPIC-MOS2 spectra. The EPIC-pn spectrum of NGC 3242 is soft, with most counts below $1.0 \mathrm{keV}$. The spectrum peaks at $0.5-0.6 \mathrm{keV}$, and then steadily declines towards higher energies. This peak is most likely due to the He-like O viI triplet at $0.57 \mathrm{keV}$.

We shall note that the shape of the EPICpn spectrum at energies below $0.55 \mathrm{keV}$ is difficult to explain. The unbinned spectrum reveals noticeable oscillations in the count rates with small number of counts in energy bins at $\sim 450$ $\mathrm{eV}$ and $\sim 500 \mathrm{eV}$, and large number of counts

\footnotetext{
3 Based on XMM-Newton observations of the bright, soft point source Nova LMC1995 (Orio et al. 2003).
} 

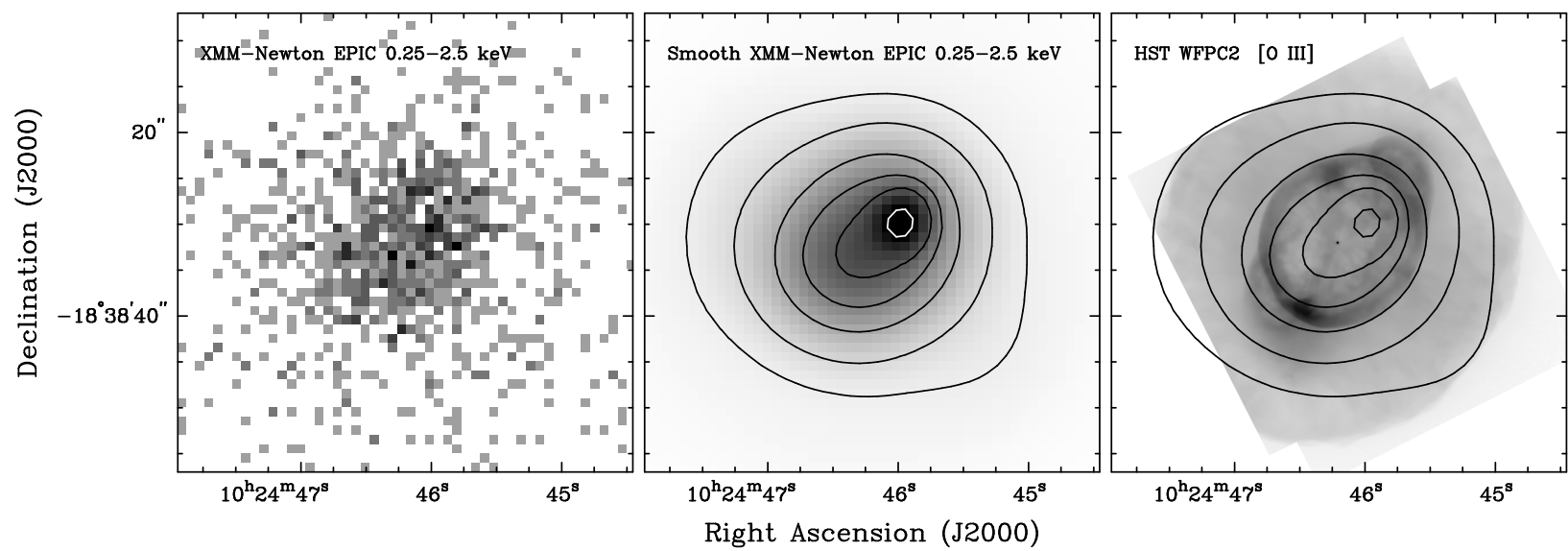

Fig. 1.- Raw (left) and smoothed (center) XMM-Newton EPIC images of NGC 3242 in the $0.25-2.5 \mathrm{keV}$ energy band, and (right) HST WFPC2-PC1 [O III] image of NGC 3242. The [O III] image is overplotted with the X-ray contours derived from the smoothed EPIC X-ray image. Contours correspond to $10 \sigma, 20 \sigma$, $50 \sigma, 75 \sigma, 100 \sigma$, and $150 \sigma$ above the background level.

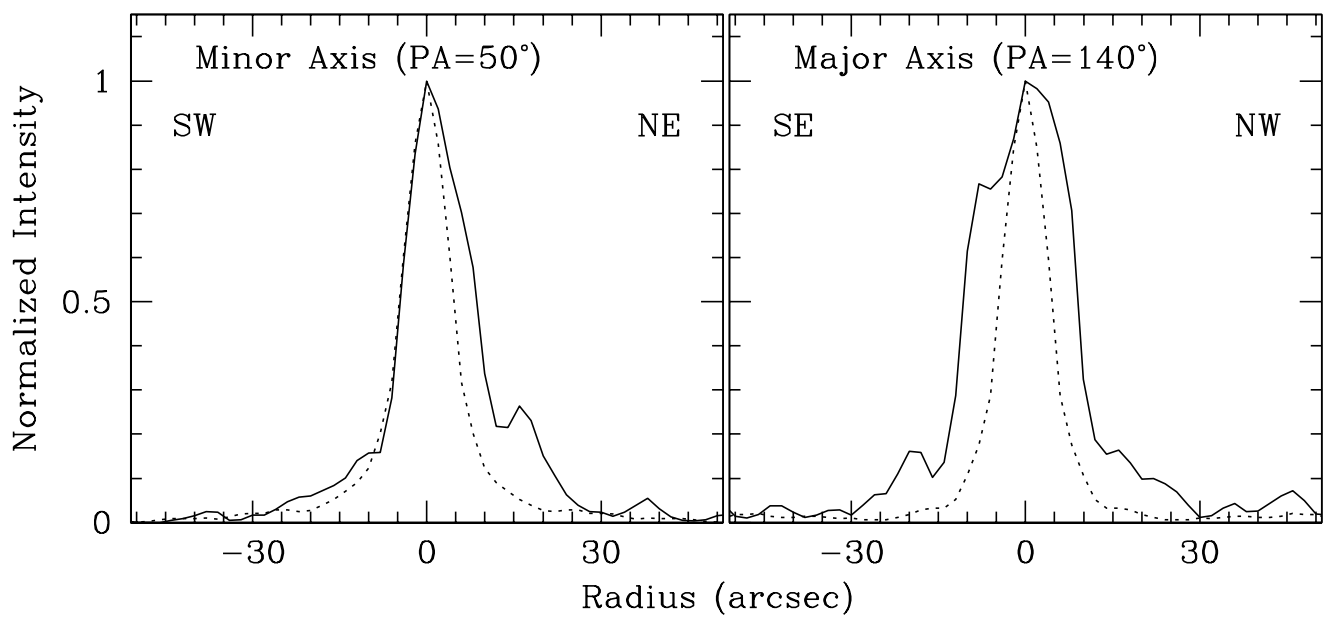

Fig. 2.- Normalized intensity of the EPIC-MOS X-ray surface brightness profile of NGC 3242 (solid line) and a point-source in the field of view of the instrument (dotted line) along the minor (left) and major (right) axes of the nebula.

in energy bins between these two energies and at $\sim 400 \mathrm{eV}$. Since these "spectral features" have widths smaller than the EPIC-pn spectral resolution at this energy range (FWHM $\sim 90 \mathrm{eV})$, it is unlikely that these spikes are associated with real emission lines. It has been reported that optical loading can produce the observed effects, but this mechanism can be ruled out because the Medium Filter used in our observations prevents the optical contamination from point sources as bright as $m_{V}=6-9$ mag. (XMM-SOC-CAL-TN0051), while the central star of NGC 3242 has $m_{V}=10.3 \mathrm{mag}$ (van Altena et al. 1995). We can conclude that these oscillations are most likely caused by stochastic effects. To mitigate this issue, the EPIC-pn spectrum has been binned to have at least 25 counts per channel for further spectral analysis. Similarly, the EPIC-MOS spectra have 


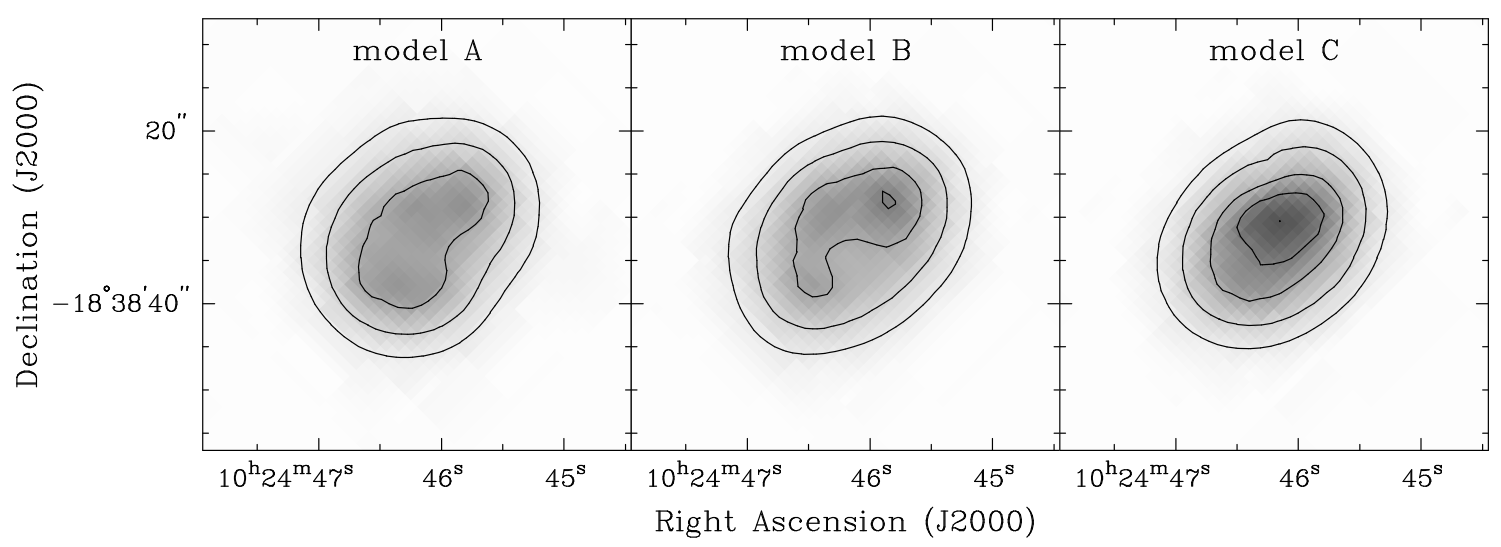

Fig. 3.- Realizations of three Monte Carlo simulations of the XMM-Newton images of NGC 3242 for an X-ray-emitting ellipsoidal shell of constant density with a shell thickness 10\% (model A) and 20\% (model $\mathrm{C}$ ), and a filled shell (model C). Contours correspond to $20 \sigma, 40 \sigma, 80 \sigma$, and $120 \sigma$ above the background level.

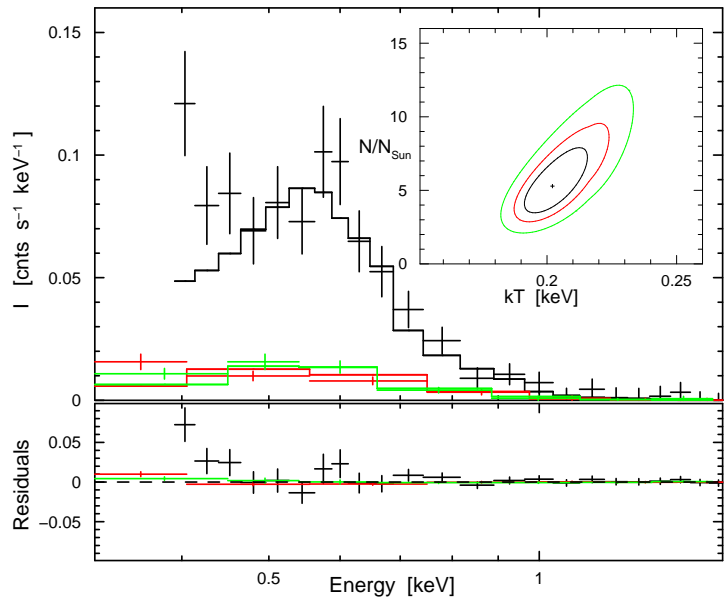

Fig. $\quad$ 4.- (top-panel) EPIC-pn (black), EPIC-MOS1 (red), and EPIC-MOS2 (green) background-subtracted spectra of the diffuse emission of NGC 3242. The best-fit joint model is shown as a histogram in the corresponding color. The inset shows the nitrogen vs. temperature $\chi^{2}$ grid plot of the spectral fit where the black, red, and green curves represent the $68 \%, 90 \%$, and 99\% confidence levels. (bottom-panel) Residuals of the best-fit joint model to the EPIC-pn (black), EPIC-MOS1 (red), and EPIC-MOS2 (green) spectra of NGC 3242 shown in the top panel. been binned to have at least 15 counts per channel for spectral analyses.

\subsection{Spectral Analysis}

For the spectral analysis, we have adopted the nebular chemical abundances $\left(\mathrm{He}=0.94 \mathrm{He}_{\odot}\right.$, $\mathrm{C}=0.78 \mathrm{C}_{\odot}, \mathrm{N}=1.61 \mathrm{~N}_{\odot}, \mathrm{O}=0.83 \mathrm{O}_{\odot}, \mathrm{Ne}=0.75 \mathrm{Ne}_{\odot}$, $\mathrm{S}=0.20 \mathrm{~S}_{\odot}$, and $\left.\mathrm{Ar}=0.40 \mathrm{Ar}_{\odot}\right)$, and foreground hydrogen column density $\left(N_{\mathrm{H}}=5 \times 10^{20} \mathrm{~cm}^{-2}\right)$ derived by Pottasch \& Bernard-Salas (2008). We have then modeled the observed EPIC spectra using an absorbed APEC optically thin plasma emission model and adopting the absorption crosssections from Morrison \& McCammon (1983).

This model provides a reasonable fit to the EPIC spectra of NGC 3242's diffuse emission with a reduced $\chi^{2}$ of $1.60(=38.4 / 24)$ for $k T=0.190 \pm 0.009$ $\mathrm{keV}\left(\sim 2.2 \times 10^{6} \mathrm{~K}\right)$, although the best-fit model is lower than the observed spectrum at energies $<0.5$ $\mathrm{keV}$. The fits are not improved by allowing $N_{\mathrm{H}}$ to vary and we find that the best-fit values of $k T$ and $N_{\mathrm{H}}$ appear to be correlated (Figure 5) as $k T=0.1975-20.0 \times N_{\mathrm{H}}$, where $k T$ is given in $\mathrm{keV}$ and $N_{\mathrm{H}}$ in units of $10^{20} \mathrm{~cm}^{-2}$.

Alternatively, we may allow the chemical abundances of nitrogen and carbon to vary in the fits, as these two elements have spectral lines that can contribute in the $0.4-0.5 \mathrm{keV}$ energy band. Variations of the carbon abundance do not produce noticeable changes in the quality of 


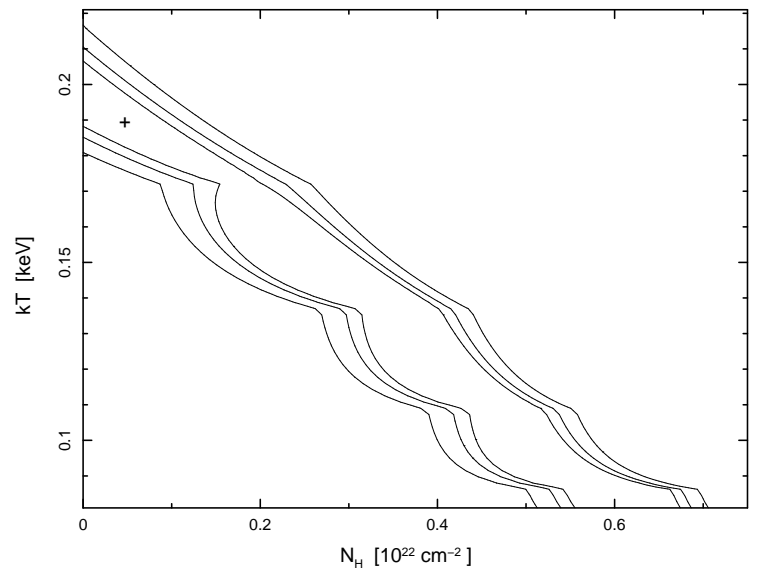

Fig. 5.- Temperature vs. column density $\chi^{2}$ grid plot of the spectral fit with nebular abundances and free column density, where the three curves represent the $68 \%, 90 \%$, and $99 \%$ confidence levels, and the cross marks the best fit for the value of $5 \times 10^{20} \mathrm{~cm}^{-2}$ adopted for $N_{\mathrm{H}}$.

the spectral fit, and thus the carbon abundance cannot be well constrained. On the other hand, changes to the nitrogen abundances produce a significant improvement of the spectral fit, and the best-fit model (Figure 4) has $k T=0.202_{-0.010}^{+0.012} \mathrm{keV}$ $\left(\sim 2.35 \times 10^{6} \mathrm{~K}\right), \mathrm{N}=5.3_{-1.8}^{+2.8} \mathrm{~N}_{\odot}$, and a reduced $\chi^{2}$ of $1.07(=24.56 / 23)$. The limits to the nitrogen abundance set by this fit are better illustrated by the nitrogen versus temperature $\chi^{2}$ grid plot of the spectral fit in Figure 4, which shows that the range of nitrogen abundances exclude the nebular abundance of $1.61 \mathrm{~N}_{\odot}$. The best-fit value for the nitrogen abundance implies a $\mathrm{N} / \mathrm{O}$ ratio of the $\mathrm{X}$ ray-emitting gas to be $(\mathrm{N} / \mathrm{O})_{\mathrm{X}} \sim 1.2, \sim 3.3$ times greater than the $\mathrm{N} / \mathrm{O}$ ratio of the ionized nebular material, $(\mathrm{N} / \mathrm{O})_{\text {neb }} \sim 0.36$.

For the best-fit model with enhanced nitrogen abundance, we derive an observed flux of $\left(4.2_{-1.1}^{+0.7}\right) \times 10^{-14} \mathrm{ergs} \mathrm{cm}^{-2} \mathrm{~s}^{-1}$ and an intrinsic Xray luminosity of $\left(7.3_{-1.3}^{+0.8}\right) \times 10^{30} d^{2} \mathrm{ergs} \mathrm{s}^{-1}$ in the $0.4-2.0 \mathrm{keV}$ energy band, where $d$ is the distance in kpc. The volume emission measure $(E M=$ $N_{\mathrm{e}}^{2} V$, where $N_{\mathrm{e}}$ is the electron density and $V$ is the emitting volume) of this model is $\sim 5 \times 10^{53} d^{2}$ $\mathrm{cm}^{-3}$. The emitting volume of the inner shell of NGC 3242 is $\sim 2 \times 10^{52} \epsilon d^{3} \mathrm{~cm}^{3}$, where $\epsilon$ is the volume filling factor of the X-ray-emitting gas. Thus, the electron density of the $\mathrm{X}$-ray-emitting gas is $\sim 5 \epsilon^{-1 / 2} d^{1 / 2} \mathrm{~cm}^{-3}$, and the thermal pressure is $1.6 \times 10^{-9} \epsilon^{-1 / 2} d^{1 / 2}$ dyne $\mathrm{cm}^{-2}$. At a distance of $0.55 \mathrm{kpc}$, the X-ray luminosity of NGC 3242 is $\left(2.2_{-0.4}^{+0.2}\right) \times 10^{30}$ ergs s$^{-1}$, the electron density of the X-ray-emitting gas is $4 \epsilon^{-1 / 2} \mathrm{~cm}^{-3}$, and its thermal pressure is $1.2 \times 10^{-9} \epsilon^{-1 / 2}$ dyne $\mathrm{cm}^{-2}$.

\section{Physical Structure of the Optical Shell}

\subsection{Bulk Physical Conditions}

The electron temperature and density of the optical shell of NGC 3242 can be derived using standard techniques (e.g., Guerrero et al. 1996) from the long-slit intermediate-dispersion spectroscopy presented in $\S 2.3$. From these two-dimensional spectra of NGC 3242, we have extracted onedimensional spectra of representative regions of its inner and outer shells, and measured the fluxes of the emission lines using the IRAF task splot. Table 2 presents line fluxes normalized to an $\mathrm{H} \beta$ flux of 100. The observed fluxes, $F$, have been dereddened using the IRAF task redcorr to derive the intrinsic intensity of the line, $I$ :

$$
I=F \times 10^{-c_{H \beta} \times f_{\lambda}}
$$

where $\mathrm{c}_{H \beta}$ is the logarithmic $\mathrm{H} \beta$ extinction constant computed by comparing the observed value of the $\mathrm{H} \alpha$ to $\mathrm{H} \beta$ ratio to the expected theoretical value of 2.87 for case $\mathrm{B}$ recombination (Osterbrock \& Ferland 2006). The observed fluxes are subsequently corrected using the values of $f_{\lambda}$ corresponding to the interstellar extinction law of Savage \& Mathis (1979). The $\mathrm{H} \alpha / \mathrm{H} \beta$ ratio measured in the medium dispersion spectra implies an extinction coefficient $c_{H \beta}=0.01$ for the inner shell and 0.05 for the outer shell. We note that these values of $c_{H \beta}$ are smaller than the values 0.10-0.15 derived by Balick et al. (1993) and Pottasch \& Bernard-Salas (2008), but consistent with the values derived by Henry et al. (2000) using this same dataset.

The physical conditions for the inner and outer shells of NGC 3242 have been derived using temperature-sensitive line ratios of [O $\mathrm{III}]$, [ $\left.\begin{array}{ll}\mathrm{N} & \mathrm{II}\end{array}\right],\left[\begin{array}{ll}\mathrm{O} & \mathrm{II}\end{array}\right]$, and $[\mathrm{S}$ III $]$, and density-sensitive line ratios of $\left[\begin{array}{ll}\mathrm{S} & \mathrm{II}\end{array}\right]$ and $[\mathrm{Ar} \mathrm{IV}]$. The ions and lines used, their ratios, and the values of electron temperature, $T_{\mathrm{e}}$, and density, $N_{\mathrm{e}}$, are listed in Table 3. The results suggest that the inner shell 
density is $\sim 2,200 \mathrm{~cm}^{-3}$, in excellent agreement with the density derived from the $[\mathrm{S}$ II] lines by Pottasch \& Bernard-Salas (2008), and the outer shell density is $\sim 370 \mathrm{~cm}^{-3}$. Among the four temperatures derived from different lines, we adopt that from the [O III] lines, 11,900 $\mathrm{K}$ for the inner shell and 10,400 K for the outer shell, as the [O III] temperature diagnostic is the least affected by the extinction law and the lines are the brightest. This radial decline in temperature is consistent with the results presented by Balick et al. (1993). Using these values, we derive thermal pressures of $3.6 \times 10^{-9}$ dyne $\mathrm{cm}^{-2}$ and $5 \times 10^{-10}$ dyne $\mathrm{cm}^{-2}$ for the inner and outer shells, respectively.

\subsection{Spatially Resolved Physical Condi- tions}

The narrow-band HST images and ratio maps of NGC 3242 in different emission lines (Figure 6) display a simple shell morphology consisting of an elliptical inner shell and an attached outer shell. This shell morphology is only moderately complicated by ansae and knots best seen in the $[\mathrm{N} \mathrm{II}]$ images (the so-called Fast Low-Ionization Emission Regions, FLIERS, Balick et al. 1998). A spatiokinematic study of NGC 3242 by Balick et al. (1987) confirms this simple shell structure: the inner shell is a nearly round bubble expanding at $25-30 \mathrm{~km} \mathrm{~s}^{-1}$, while the outer shell is a coexpanding envelope filled with material. Thus, the $\mathrm{H} \alpha$ image of NGC 3242 can be used to obtain density and pressure profiles of the nebula for comparison with those of the X-ray-emitting gas in the central cavity.

The density profile of NGC 3242 has been previously determined by Soker et al. (1992) using a ground-based $\mathrm{H} \alpha$ image. We will use the highresolution HST WFPC2 $\mathrm{H} \alpha$ image to determine the electron density, $N_{\mathrm{e}}$, and thermal pressure, $P_{\mathrm{th}}$, of the inner shell and the envelope as a function of nebular radius. First, we examined this image to select a direction that would provide us with a clean surface brightness profile. The cut along $\mathrm{PA}=230^{\circ}$ is orthogonal to the inner shell rim and covers the full extent of the outer shell. Thus, we extracted the surface brightness profiles in the $\mathrm{H} \alpha$, He II $\lambda 4686$, [O III] $\lambda 5007$, and [N II] $\lambda 6583$ shown in Figure 7 . The surface brightness profile of the outer shell is very similar in the $\mathrm{H} \alpha$, [O III], and [N II] lines, but the He II emission is

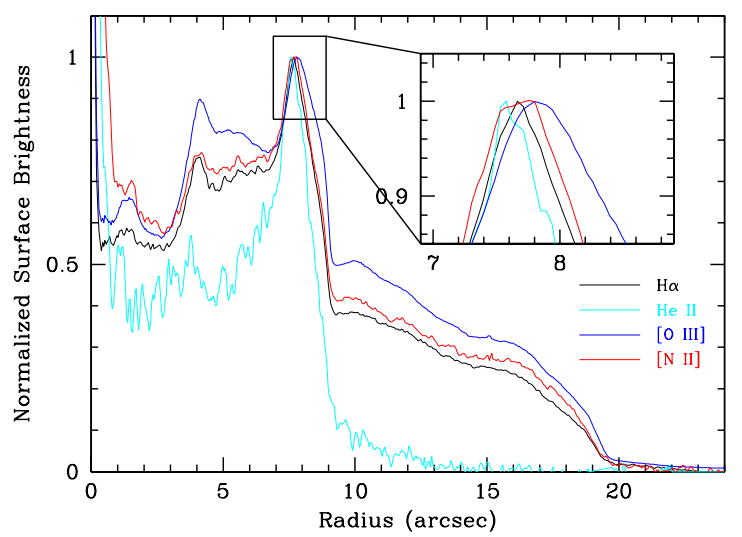

Fig. 7.- $\mathrm{H} \alpha$, He II $\lambda 4686$, [O III] $\lambda 5007$, and [N II] $\lambda 6583$ surface brightness profiles of the inner and outer shells of NGC 3242 normalized to the bright peak at the rim of the inner shell. The profiles are derived by averaging the surface brightness in radial bins in a sector along $\mathrm{PA}=230^{\circ}$ with $\Delta \mathrm{PA}$ $=10^{\circ}$. The inset expands this peak to better illustrate the spatial differences at this location of the emission in the different lines.

diminished most likely because the inner shell is optically thick to $\mathrm{He}^{+}$ionizing photons. As for the inner shell, all profiles show the bright shell rim, but peak at different radial distances: $\sim 7^{\prime \prime} 4$ for He II, $\sim 7^{\prime \prime} .6$ for $\mathrm{H} \alpha$, and $\sim 7^{\prime \prime} .8$ for [O III]. The emission inside this rim drops steeply, but the filaments projected in the central cavity produce secondary peaks in the $\mathrm{H} \alpha,[\mathrm{O}$ III $]$, and [N II] lines, but not in the He II line.

The outer shell has been modeled assuming a mean electron density $\sim 370 \mathrm{~cm}^{-3}$ and a temperature of $10,400 \mathrm{~K}$. Figure 8 left shows the fit to the $\mathrm{H} \alpha$ surface brightness profile of the outer shell of NGC 3242 assuming four different radial dependences of the density: $r^{-2}, r^{-1}, r^{-1 / 2}$, and $r^{-1 / 3}$. The model surface brightnesses for the $r^{-2}$ and $r^{-1}$ density profiles decrease outwards too rapidly compared to the observed surface brightness profile, and can thus be excluded. The model surface brightness for the $r^{-1 / 2}$ and $r^{-1 / 3}$ density profiles more closely match the observation.

Using the $r^{-1 / 2}$ density profiles in the outer shell, we proceed to model the $\mathrm{H} \alpha$ and $\mathrm{He}$ II surface brightness profiles at the rim of the inner shell by varying the thickness of the shell and ad- 

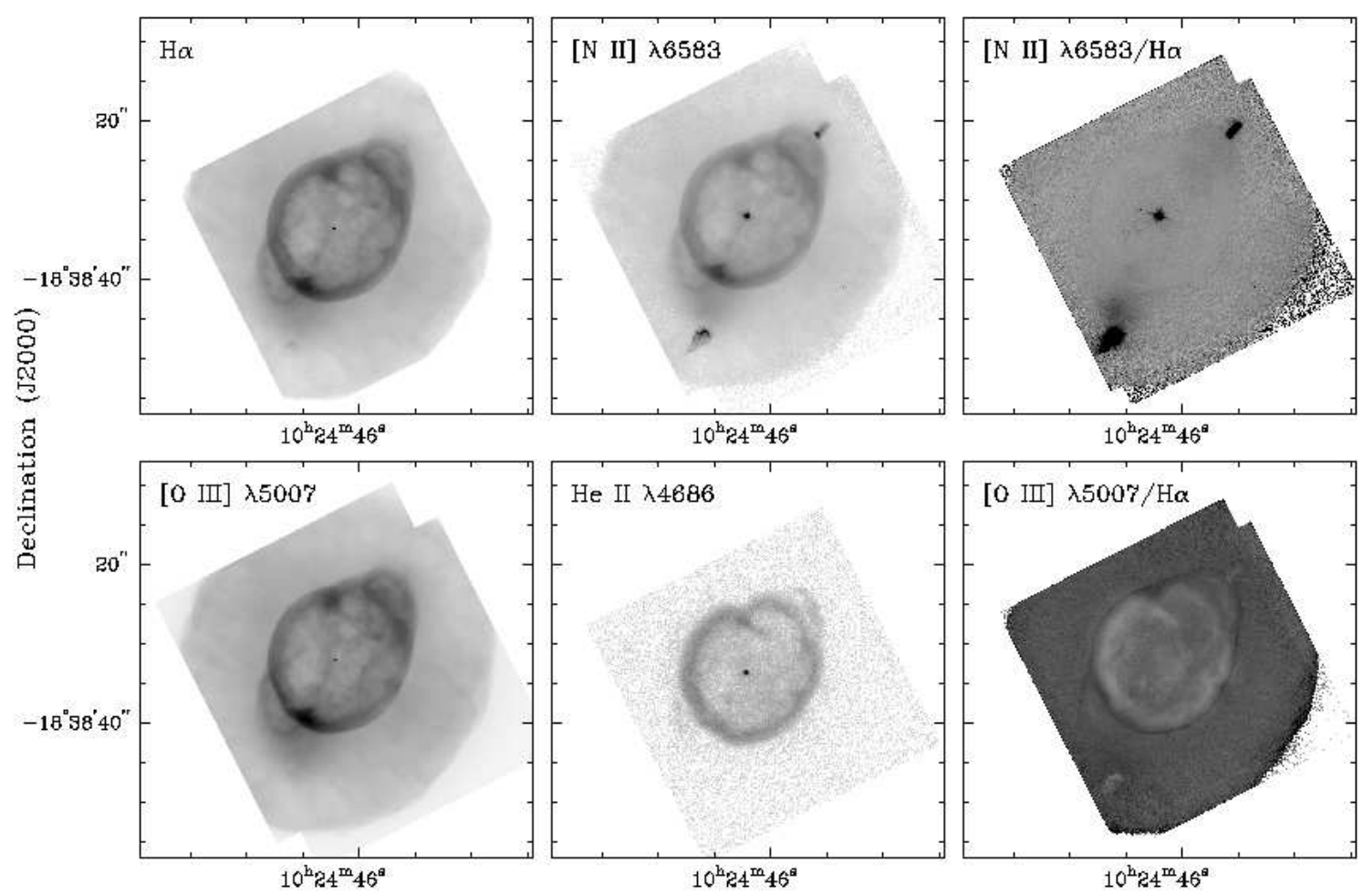

Right Ascension (J2000)

Fig. 6.- HST WFPC2 narrow-band images and ratio maps of the central region of NGC 3242. The images are displayed on a squared-root scale, while the ratio maps are displayed on a linear scale.

justing the ionization fraction of $\mathrm{He}^{++}$. A shell with a constant density $2,200 \mathrm{~cm}^{-3}$, temperature of $11,900 \mathrm{~K}$, shell thickness $\sim 15 \%$, and a sharp decay in its inner edge produces a good fit to the He II profile. The fit to the rim of the shell in the $\mathrm{H} \alpha$ profile is also reasonable, but the model profile does not include the contributions from filaments to the $\mathrm{H} \alpha$ profile (Figure 8 right).

The density profile deduced from the model comparisons is shown in Figure 9-left. The radial profile of the thermal pressure (Figure9-right) has been constructed assuming a constant electron temperature of $11,900 \mathrm{~K}$ for the inner shell, and $10,400 \mathrm{~K}$ for the outer shell, as derived from temperature sensitive optical line ratios. This figure shows that the thermal pressure of the inner shell is much higher than that of the outer shell, i.e., the inner shell is expanding into the envelope.

We can also compare the thermal pressure of the nebular shell to that of the hot interior.
Assuming that the hot gas is distributed in a constant-density shell interior to but in contact with the inner nebular shell, the filling factor can be expressed as $\epsilon=1-\left(r_{i} / r_{o}\right)^{3}$, where $r_{o}$ and $r_{i}$ are the outer and inner radii of the hot gas shell $\left(r_{o}\right.$ is also the inner radius of the inner nebular shell). As the hot gas density is $\sim 4 \epsilon^{-1 / 2} \mathrm{~cm}^{-3}(d=0.55$ $\mathrm{kpc}$ ), we have plotted the hot gas density as a function of its inner shell radius $r_{i}$ in Figure 9-left and the thermal pressure in Figure 9-right. The hot gas shell's inner radii corresponding to $\epsilon$ of $0.15,0.5$, and 0.8 are marked. It is apparent that the hot gas pressure exceeds that of the nebular shell only for small filling factor values, $\epsilon<0.15$, thus requiring the hot gas to be concentrated in a thin shell with fractional thickness $\lesssim 0.05$.

\section{Discussion}

The properties of the X-ray emission from NGC 3242, based on a preliminary analysis (Ruiz et al. 

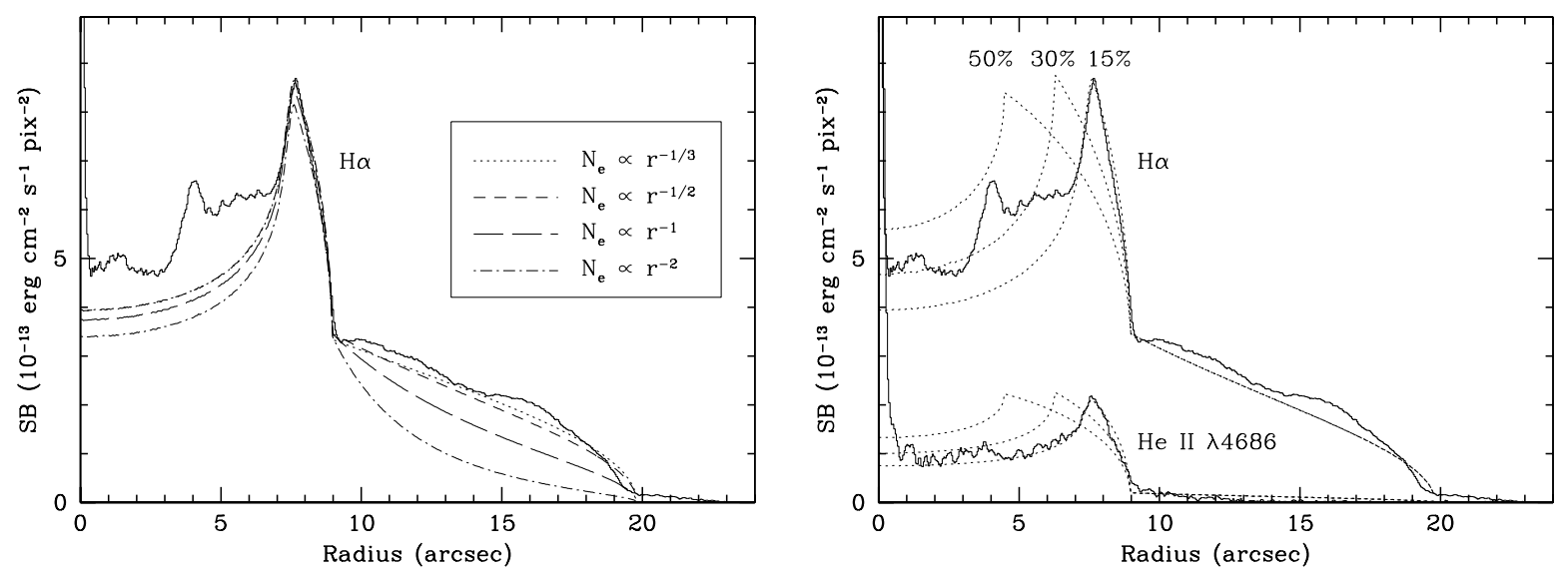

Fig. 8.- (left) $\mathrm{H} \alpha$ surface brightness profile of NGC 3242 along $\mathrm{PA}=230^{\circ}$ (thick line) and synthetic $\mathrm{H} \alpha$ surface brightness profiles of the outer shell $(r>8$ ". 9$)$ for different radial dependences of $N_{\mathrm{e}}$. As for the inner shell, a thickness of $15 \%$ in radius has been assumed (see below). (right) $\mathrm{H} \alpha$ and He II $\lambda 4686$ surface brightness profile of NGC 3242 along $\mathrm{PA}=230^{\circ}$ (thick lines) and synthetic surface brightness profiles of the inner shell for different values of the shell thickness: $15 \%, 30 \%$, and $50 \%$. As for the outer shell, a decay of $N_{\mathrm{e}} \propto r^{-1 / 2}$ has been assumed (see above).
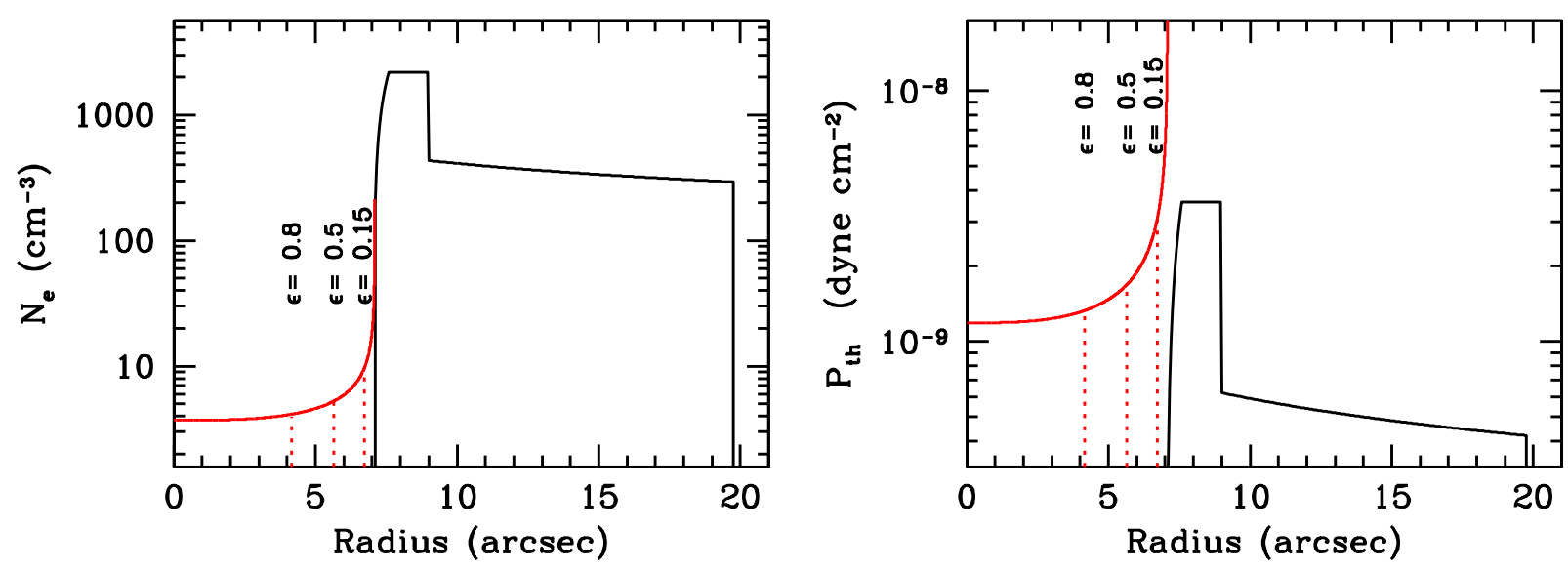

Fig. 9.- (left) Electron density, $N_{\mathrm{e}}$, and (right) thermal pressure, $P_{\mathrm{th}}$, radial profiles of the central cavity and the inner shell and envelope of NGC 3242. The density and thermal pressure of the X-ray-emitting gas within the central cavity (radius $\lesssim 7^{\prime \prime}$ ) are shown as a function of the filling factor, $\epsilon$ (red curves). The values of these physical conditions for filling factors, $\epsilon$, of $0.15,0.5$, and 0.8 are further labeled on the plot at the inner radii of the corresponding hypothetical hot gas shell.

2006), were compared to 1D hydrodynamical models of PNe that included the effects of stellar wind evolution and heat conduction by Steffen et al. (2008). They remarked that the round shape of the rim of the inner shell of NGC 3242 makes it a suitable $\mathrm{PN}$ for the comparison with their 1D simulations. The revised values of different Xray properties of NGC 3242 presented here need 
to be discussed in the framework of Steffen et al. (2008)'s model. This revision mostly affects the values of the thermal pressure of the hot bubble and inner shell rim, whereas the X-ray luminosity (scaled to the distance of $0.55 \mathrm{kpc}$ ) and plasma temperature of NGC 3242 are basically the same as those used by Steffen et al. (2008).

We note that the models selected by Steffen et al. (2008) have a thermal pressure of the hot bubble that exceeds that of the rim, while our revised values indicate the opposite, i.e., the thermal pressure of the rim $\left(P_{\mathrm{th}}=3.6 \times 10^{-9}\right.$ dyne $\left.\mathrm{cm}^{-2}\right)$ is higher than that of the bubble $\left(P_{\mathrm{th}}=1.6 \times 10^{-9} \epsilon^{-1 / 2} d^{1 / 2}\right.$ dyne $\mathrm{cm}^{-2}$ ). One possible outcome to this issue would be a distance for NGC 3242 much farther than $0.55 \mathrm{kpc}$ (Terzian 1997; Mellema 2004); at a distance of $3 \mathrm{kpc}$, the thermal pressures of the hot bubble and rim would be the same, but this would make NGC 3242 too large $(r=0.12 \mathrm{pc})$ and evolved ( $\tau=6,000 \mathrm{yrs})$ and we consider it to be unlikely.

The other possible solution is to consider a low value for the filling factor of the $\mathrm{X}$-ray-emitting gas in the bubble; a value $\epsilon=0.15$ would make both pressures the same. Such a low filling factor is expected to produce a noticeable limb-brightening morphology that is not observed (Figures 1 and 2). As shown in Fig. 3, hints of a limb-brightened morphology are still apparent for a hot gas shell with a fractional thickness of 0.2 (i.e., $\epsilon \sim 0.5$ ) even at the $X M M-N e w t o n$ limited spatial resolution $\left(\sim 15^{\prime \prime}\right)$. It is arguable, however, that extinction has reduced the center-to-limb contrast of the X-ray emission, thus shifting the emission inwards, as this is an effect expected to be noticeable (Steffen et al. 2008) even for the small extinction $\left(N_{\mathrm{H}}=5 \times 10^{20} \mathrm{~cm}^{-2}\right)$ towards the nebula.

Alternatively, we must consider the dynamical effects of the photo-ionization of the nebular material, which may have overcome those of the currently diminished stellar wind of NGC 3242 (Kudritzki et al. 1997; Tinkler \& Lamers 2002). In this case, the nebular expansion is driven by the thermal pressure increase produced in the rim by photo-ionization. This may explain the relatively large thickness of NGC 3242 inner shell rim, as compared to that of NGC 6543. We suggest that the rim thickness can be used as an indicator of the relative thermal pressure of hot gas and photo-ionized nebular material; a sharp rim would imply that the thermal pressure of the hot gas is larger than this of the nebula, and thus that hot gas itself is present (and detectable), while a thick inner shell rim would imply a lower pressure for the hot gas, if present.

The low plasma temperature is puzzling in view of the enhanced $\mathrm{N} / \mathrm{O}$ ratio of the X-rayemitting plasma, about three times higher than the $\mathrm{N} / \mathrm{O}$ ratio of the nebular material. Such differences in the chemical abundances seem to imply that the X-ray-emitting plasma mostly consists of shocked stellar wind, with little contamination of material from the nebula. The low plasma temperature, however, needs large amounts of material from the cold nebular shell to have been incorporated into the hot bubble. This problem, similar to that found in other PNe such as BD+30³639 (Yu et al. 2009), NGC 2392 (Guerrero et al. 2005), and NGC 6543 (Chu et al. 2001), may require mechanisms for the production of X-ray emission in PNe other than mixing and heat conduction as summarized by Soker et al. (2010).

There is, finally, a noticeable asymmetry of the spatial distribution of the X-ray emission from NGC 3242, with the northwestern half of the hot bubble being brighter than its southeastern half (Figures 1 and 2). Even if we assume that the inner shell of NGC 3242 were a tilted ellipsoid with its Northwestern tip moving towards us (and its Southeastern tip receding from us), it seems unlikely that an uneven spatial distribution of intervening material could be responsible of the observed asymmetry as it may be the case of the dusty $\mathrm{PNe} \mathrm{BD}+30^{\circ} 3639$ (Kastner et al. 2000) and NGC 7027 (Kastner et al. 2001), because only small extinction variations were observed in NGC 3242 (Balick et al. 1993). On the other hand, our simulations in Section 3.1 suggest that the asymmetric distribution of X-ray emission could result from small number statistics.

\section{Summary}

We have obtained $X M M-N e w t o n$ X-ray observations of NGC 3242, a multiple-shell PN consisting of an inner shell with a bright, round rim, and an outer envelope. The observations have detected diffuse, soft X-ray emission confined within the innermost shell of NGC 3242. The relatively low 
temperature of the hot gas, $T_{X}=2.35 \times 10^{6} \mathrm{~K}$, compared to the expected adiabatic post-shock temperature of a stellar wind with a velocity of 2,400 $\mathrm{km} \mathrm{s}^{-1}$ (Pauldrach et al. 2004) suggests that heat conduction has taken place. Indeed, models including heat conduction provide a reasonable description of the X-ray temperature and luminosity of NGC 3242 (Steffen et al. 2008). However, the chemical abundances of the X-ray-emitting plasma are closer to the stellar values, suggesting little evaporation of cold nebular material into the hot bubble and thus contradicting the expectation of heat conduction.

We have compared the physical properties $\left(N_{e}\right.$, $\left.T_{e}, P_{\mathrm{th}}\right)$ of the gas in the hot bubble with those of the gas at the inner shell rim. The inner shell can be described as a thin shell with a constant density of $2,200 \mathrm{~cm}^{-3}$ and a thickness $15 \%$ its radius, while the envelope is best described by a shell whose density declines $\propto \mathrm{r}^{-1 / 2}$ to $\mathrm{r}^{-1 / 3}$. The gas in the hot bubble has lower thermal pressure than the gas in the shell rim, unless the X-ray-emitting gas is mostly confined within a thin shell, which is not supported by the X-ray morphology observed. Comparisons with simulations favor a large filling factor for the X-ray-emitting gas.

We also note the asymmetric distribution of the X-ray emission within the inner shell of NGC 3242 and found it unlikely to be a result of nonuniform absorption of the X-ray emission within the optical shell. X-ray observations at higher spatial resolution and signal-to-noise ratio (Montez et al., in preparation) are needed to provide a sharper view of the distribution of the X-ray-emitting plasma within NGC 3242.

We thank the anonymous referee for comments that helped us to improve this paper. N.R. and M.A.G. are partially funded by grant AYA 200801934 of the Spanish MICINN (Ministerio de Ciencia e Innovación). Y.-H.C. and R.A.G acknowledge the support of NASA grant NNG04GE63G for this research project. We are also very grateful to Dr. Karen Kwitter for providing us with the optical long-slit spectra of NGC 3242. Some of the data presented in this paper were obtained from the Multimission Archive at the Space Telescope Science Institute (MAST). STScI is operated by the Association of Universities for Research in Astronomy, Inc., under NASA contract NAS5-26555.
Facilities: HST(WFPC), XMM, KPNO $(2.1 \mathrm{~m}$ telescope).

\section{REFERENCES}

Balick, B., Alexander, J., Hajian, A. R., Terzian, Y., Perinotto, M., \& Patriarchi, P. 1998, AJ, 116,360

Balick, B., Preston, H. L., \& Icke, V. 1987, AJ, 94, 1641

Balick, B., Rugers, M., Terzian, Y., \& Chengalur, J. N. 1993, ApJ, 411, 778

Cerruti-Sola, M., \& Perinotto, M. 1985, ApJ, 291, 237

Chu, Y.-H., Guerrero, M. A., Gruendl, R. A., Williams, R. M., \& Kaler, J. B. 2001, ApJ, 553, L69

Corradi, R. L. M., Schönberner, D., Steffen, M., \& Perinotto, M. 2003, MNRAS, 340, 417

Corradi, R. L. M., Sánchez-Blázquez, P., Mellema, G., Giammanco, C., \& Schwarz, H. E. 2004, A\&A, 417, 637

Eder, J., Lewis, B. M., \& Terzian, Y. 1988, ApJS, 66,183

Guerrero, M. A., Chu, Y.-H., Gruendl, R. A., \& Meixner, M. 2005, A\&A, 430, L69

Guerrero, M. A., Gruendl, R. A., \& Chu, Y.-H. 2002, A\&A, 387, L1

Guerrero, M. A., Chu, Y.-H., Gruendl, R. A., Williams, R. M., \& Kaler, J. B. 2001, ApJ, 553, L55

Guerrero, M. A., Chu, Y.-H., \& Gruendl, R. A. 2000, ApJS, 129, 295

Guerrero, M. A., Manchado, A., \& Serra-Ricart, M. 1996, ApJ, 456, 651

Guerrero, M. A., Ramos-Larios, G., \& Massa, D. 2010, PASA, 27, 210

Henry, R. B. C., Kwitter, K. B., \& Bates, J. A. 2000, ApJ, 531, 928

Kastner, J. H., Balick, B., Blackman, E. G., Frank, A., Soker, N., Vrtílek, S. D., \& Li, J. 2003, ApJ, 591, L37 
Kastner, J. H., Soker, N., Vrtílek, S. D., \& Dgani, R. 2000, ApJ, 545, L57

Kastner, J. H., Vrtílek, S. D., \& Soker, N. 2001, ApJ, 550, L189

Kudritzki, R. P., Méndez, R. H., Puls, J., \& McCarthy, J. K. 1997, Planetary Nebulae, 180, 64

Kwok, S. 1983, Planetary Nebulae, 103, 293

Mellema, G., \& Frank, A. 1995, MNRAS, 273, 401

Mellema, G. 2004, A\&A, 416, 623

Montez, R., De Marco, O., Kastner, J. H., \& Chu, Y.-H. 2010, ApJ, 721, 1820

Morrison, R., \& McCammon, D. 1983, ApJ, 270, 119

Orio, M., Hartmann, W., Still, M., \& Greiner, J. 2003, ApJ, 594, 435

Osterbrock, D. E., \& Ferland, G. J. 2006, Astrophysics of gaseous nebulae and active galactic nuclei, 2nd. ed. by D.E. Osterbrock and G.J. Ferland. Sausalito, CA: University Science Books, 2006

Pauldrach, A. W. A., Hoffmann, T. L., \& Méndez, R. H. 2004, A\&A, 419, 1111

Pottasch, S. R., \& Bernard-Salas, J. 2008, A\&A, 490, 715

Ruiz, N., Guerrero, M. A., Chu, Y.-H., Gruendl, R. A., Kwitter, K. B., \& Meixner, M. 2006, Planetary Nebulae in our Galaxy and Beyond, 234, 497

Savage, B. D., \& Mathis, J. S. 1979, ARA\&A, 17, 73

Soker, N., \& Kastner, J. H. 2003, ApJ, 583, 368

Soker, N., Rahin, R., Behar, E., \& Kastner, J. H. 2010, ApJ, 725, 1910

Soker, N., Zucker, D. B., \& Balick, B. 1992, AJ, 104,2151

Steffen, M., Schönberner, D., \& Warmuth, A. 2008, A\&A, 489, 173

Terzian, Y. 1997, Planetary Nebulae, 180, 29
Tinkler, C. M., \& Lamers, H. J. G. L. M. 2002, A\&A, 384, 987

van Altena, W. F., Lee, J. T., \& Hoffleit, D. 1995, VizieR Online Data Catalog, 1174, 0

Weaver, R., McCray, R., Castor, J., Shapiro, P., \& Moore, R. 1977, ApJ, 218, 377

Yu, Y. S., Nordon, R., Kastner, J. H., Houck, J., Behar, E., \& Soker, N. 2009, ApJ, 690, 440

Zhekov, S. A., \& Perinotto, M. 1998, A\&A, 334, 239

This 2-column preprint was prepared with the AAS LATEX macros v5.2. 
TABle 1

HST WFPC2 Observations of NGC 3242

\begin{tabular}{lccl}
\hline \hline Emission Lines & Number of images & $\begin{array}{c}t_{\text {exp }} \\
{[\mathrm{s}]}\end{array}$ & Program ID \\
\hline $\mathrm{H} \alpha$ & 1 & 100 & 6117 \\
$\mathrm{He}$ II & 1 & 160 & 6117 \\
{$[\mathrm{~N} \mathrm{II}]$} & 3 & 400 & 6117 \\
& 2 & 300 & 7501,8773 \\
{$[\mathrm{O}$ III] } & 4 & 260 & 7501,8773 \\
& 1 & 260 & 7501 \\
& 5 & 200 & 7501,8773 \\
\hline
\end{tabular}

TABLE 2

Line StRengths For NGC 3242

\begin{tabular}{|c|c|c|c|c|c|c|}
\hline \multirow[b]{2}{*}{ Line ID } & \multirow[b]{2}{*}{$\begin{array}{c}\text { Wavelength } \\
(\AA)\end{array}$} & \multirow[b]{2}{*}{$f_{\lambda}$} & \multicolumn{2}{|c|}{ Inner Shell } & \multicolumn{2}{|c|}{ Outer Shell } \\
\hline & & & $F$ & $I$ & $F$ & $I$ \\
\hline$\left[\begin{array}{ll}\mathrm{O} & \mathrm{II}\end{array}\right]$ & 3726 & 0.26 & 10.8 & 11.7 & 3.7 & 4.0 \\
\hline$\left[\begin{array}{ll}\mathrm{O} & \mathrm{II}\end{array}\right]$ & 3729 & 0.26 & 9.7 & 10.5 & 0.3 & 0.3 \\
\hline$\left[\begin{array}{ll}\mathrm{S} & \mathrm{II}\end{array}\right]$ & 4068 & 0.20 & 0.5 & 0.5 & $\ldots$ & $\ldots$ \\
\hline$\left[\begin{array}{ll}\mathrm{S} & \mathrm{II}\end{array}\right]$ & 4076 & 0.20 & 0.5 & 0.5 & $\ldots$ & $\ldots$ \\
\hline $\mathrm{H} \delta$ & 4101 & 0.18 & 26.4 & 25.6 & 27.2 & 25.5 \\
\hline $\mathrm{H} \gamma$ & 4340 & 0.135 & 45.5 & 47.0 & 46.2 & 48.2 \\
\hline$[\mathrm{O}$ III] & 4363 & 0.13 & 10.9 & 11.3 & 10.1 & 10.5 \\
\hline [Ar IV] & 4711 & 0.04 & 4.8 & 4.9 & $\begin{array}{r}10.1 \\
4.0\end{array}$ & 4.0 \\
\hline [Ar IV] & 4740 & 0.04 & 4.0 & 4.0 & 2.9 & 2.9 \\
\hline $\mathrm{H} \beta$ & 4861 & 0.00 & 100.0 & 100.0 & 100.0 & 100.0 \\
\hline$\left[\begin{array}{ll}\mathrm{O} & \mathrm{III}\end{array}\right]$ & 4959 & -0.02 & 339.1 & 336.8 & 362.2 & 359.7 \\
\hline [O III] & 5007 & -0.03 & 1018.1 & 1007.8 & 1523.3 & 1507.9 \\
\hline$\left[\begin{array}{ll}\mathrm{Cl} & \mathrm{IIII}\end{array}\right]$ & 5517 & -0.15 & 0.2 & 0.2 & 0.3 & 0.3 \\
\hline$\left[\begin{array}{lll} & \mathrm{N} & \mathrm{II}\end{array}\right]$ & 5755 & -0.21 & 0.07 & 0.07 & $\ldots$ & $\ldots$ \\
\hline$[\mathrm{S}$ III] & 6312 & -0.30 & 0.7 & 0.6 & 0.4 & 0.4 \\
\hline$\left[\begin{array}{ll}\mathrm{N} & \mathrm{II}\end{array}\right]$ & 6548 & -0.34 & 0.7 & 0.6 & $\ldots$ & $\ldots$ \\
\hline $\mathrm{H} \alpha$ & 6563 & -0.34 & 289 & 287 & 296 & 286 \\
\hline$[\mathrm{N}$ II $]$ & 6583 & -0.34 & 2.1 & 1.9 & 14.6 & 13.4 \\
\hline$\left[\begin{array}{ll}\mathrm{S} & \mathrm{II}\end{array}\right]$ & 6716 & -0.36 & 0.3 & 0.3 & 1.1 & 1.0 \\
\hline$\left[\begin{array}{ll}\mathrm{S} & \mathrm{II}\end{array}\right]$ & 6731 & -0.36 & 0.4 & 0.4 & 1.0 & 0.9 \\
\hline$\left[\begin{array}{ll}\mathrm{O} & \mathrm{II}\end{array}\right]$ & 7320 & -0.43 & 0.5 & 0.4 & 0.05 & 0.04 \\
\hline$\left[\begin{array}{ll}\mathrm{O} & \mathrm{II}\end{array}\right]$ & 7330 & -0.43 & 0.5 & 0.4 & 0.05 & 0.04 \\
\hline$[\mathrm{S}$ III] & 9069 & -0.64 & 3.7 & 3.1 & 22.6 & 19.2 \\
\hline$[\mathrm{S}$ III $]$ & 9532 & -0.65 & 18.0 & 15.2 & 18.1 & 15.2 \\
\hline $\mathrm{F}(\mathrm{H} \beta)\left(\operatorname{ergs~cm}{ }^{-2} \mathrm{~s}^{-1}\right)$ & & & $3.1 \times 10^{-11}$ & & $2.2 \times 10^{-12}$ & \\
\hline
\end{tabular}

TABLE 3

Physical Conditions in NGC 3242

\begin{tabular}{|c|c|c|c|c|c|c|}
\hline \multirow[b]{2}{*}{ Physical Parameter } & \multirow[b]{2}{*}{ Ion } & \multirow[b]{2}{*}{ Line Ratios } & \multicolumn{2}{|c|}{ Observed Ratios } & \multicolumn{2}{|c|}{ Value } \\
\hline & & & Inner Shell & Outer Shell & Inner Shell & Outer Shell \\
\hline$T_{e}$ & {$[\mathrm{~N}$ II $]$} & $(6548+6583 / 5755$ & 41.6 & & $14,700 \mathrm{~K}$ & \\
\hline$T_{e}$ & {$[\mathrm{~S}$ III $]$} & $(9069+9532) / 6312$ & 32.0 & 106.6 & $14,040 \mathrm{~K}$ & $8,070 \mathrm{~K}$ \\
\hline$T_{e}$ & {$[\mathrm{O}$ III $]$} & $(4959+5007) / 4363$ & 124.9 & 189.0 & $11,880 \mathrm{~K}$ & $10,400 \mathrm{~K}$ \\
\hline$T_{e}$ & {$[\mathrm{O}$ II $]$} & $(3726+3729) /(7320+7330)$ & 19.5 & 43.3 & $10,100 \mathrm{~K}$ & $9,860 \mathrm{~K}$ \\
\hline$N_{e}$ & [Ar IV] & $4711 / 4740$ & 1.18 & 1.37 & $2,250 \mathrm{~cm}^{-3}$ & $400 \mathrm{~cm}^{-3}$ \\
\hline$N_{e}$ & {$\left[\begin{array}{ll}\mathrm{S} & \mathrm{II}\end{array}\right]$} & $6716 / 6731$ & 0.71 & 1.13 & $2,200 \mathrm{~cm}^{-3}$ & $340 \mathrm{~cm}^{-3}$ \\
\hline
\end{tabular}

